\title{
Performance Evaluation Of SVM and ANN for Cancer Classifi- cation and Designing Algorithm for Arrhythmia Prediction in Cancer Patient
}

\author{
Chaithanya Lakshmi ${ }^{1 *}$, Sachin Bojewar ${ }^{2}$, \\ ${ }^{1}$ Department of Information Technology, Vidyalankar Institute of Technology, Mumbai \\ ${ }^{2}$ Department of Information Technology, Vidyalankar Institute of Technology, Mumbai \\ *Corresponding author E-mail: chaithu.shastry@gmal.com
}

\begin{abstract}
Classifying the cancer based on the age and predicting the arrhythmia in cancer patient is necessary to determine the next steps in dealing with the patients. This prediction can be done by using multiple algorithms of machine learning such as SVM, Linear classifier, neural network. Machine learning, interpretability refers to understand the underlying behaviour of the prediction of a model in order to identify diagnosis criteria and/or new rules from its output. Interpretability contributes to increase the usability of the method. Also, it is relevant in decision support systems, such as in medical applications. Using multiple algorithm on big data set and predicting the arrhythmia cases from early age to old age.

Apache (Acute Physiology, Age and Chronic Health Evaluation) and SOFA (Sequential Organ Failure Assessment) score are the important factor in critically ill patients. The number of ICU (intensive care unit) admission will be depending on these two scores. Analyzing Apache and SOFA scores will be helpful for intensivist.[4]
\end{abstract}

Keywords: SVM, Neural Network, Classification, arrhythmia, Apache and SOFA score

\section{Introduction}

Cancer and arrhythmia are deadliest disease in the world. According to medical survey, each year millions of people discover they have cancer and millions of people die from the disease. However, evidence shows that 30 - 40 percent of these deaths can be prevented, and one-third can be cured through early diagnosis and treatment.

Cardiac arrhythmias are generally produced by one of three mechanisms: enhanced automaticity, triggered activity, or reentry. Reentry, which occurs when a propagating impulse fails to die out after normal activation of the heart and persists to re-excite the heart after the refractory period has ended, is the electrophysiologic mechanism responsible for the majority of clinically important arrhythmias. [8]

If arrhythmia attacks to cancer patient they have to get admitted to ICU (intensive care unit), it is intensivists responsibility to counsel the patient and their relatives. Prediction of arrhythmia in cancer patients will be helpful for an intensivist.

\section{Purpose of Work}

In the existing system, there are many types of cancers are classified using machine learning. Majorly the cancer classification is made based on tissue type, classification by grade, classification by stage. Current research is going on classification by gene. In the collected dataset there are many different types of cancer are present. Classifying the dataset based on early age to old age, will help to predict the arrhythmia in all age category. That prediction will helpful for doctors while counselling the patient and their relatives. Usually the critically ill patients are get admitted to the ICU (intensive care unit). This ICU admission days are depending on patient condition and different parameters. But the doctors mainly look the Apache (Acute Physiology, Age and Chronic Health Evaluation) and SOFA (Sequential Organ Failure Assessment) score and decides the number of ICU admission days. So prediction of number of ICU admission days will be helpful for doctors to decide the patient admission in the ICU.

Data normalization is applying on gathering data and make it in form which is required as an input to the SVM (Support Vector Machine) and ANN (Artificial Neural Network). The data set will be trained and tested using algorithm and unknown data set also be tested to calculate the accuracy of the algorithm.

After classification method, the arrhythmia cases are predicted in early age to old age. And the number of ICU admission days will be predicted based on the Apache and SOFA score of the patient. The system gives the accuracy evaluation of two algorithms. In that it shows which gives the better accuracy of the prediction of arrhythmia cases and prediction of number of ICU admission days.

\section{Findings from Study}

Cancer is classified in different ways, for example classified based on tissue types, grades, stages and latest research is going on classification by genes[5]. Majorly, cancer images are classified using SVM and ANN algorithm. Cancer data is also classified using same algorithms.

\subsection{Related Work}


Cancer classification will be helpful for doctors to counsel the patient and their relatives and it will be helpful for data analysist for analyses the cancer data for further study. Cancer classification is done using SVM, ANN and Fuzzy c-means algorithm. Major work related to cancer classification using different types of cancer images, few are listed in the table given below.

Table 3.1 cancer classification using images

\begin{tabular}{|l|l|l|l|}
\hline Sr.No & Paper title & algorithm & $\begin{array}{l}\text { Cancer } \\
\text { type }\end{array}$ \\
\hline 1 & $\begin{array}{l}\text { The Application Of Wavelet Recur- } \\
\text { rent Neural } \\
\text { Network/or Lung Cancer Classifica- } \\
\text { tion [2] }\end{array}$ & $\begin{array}{l}\text { wavelet } \\
\text { Recurrent } \\
\text { neural net- } \\
\text { work, }\end{array}$ & $\begin{array}{l}\text { Lung } \\
\text { cancer }\end{array}$ \\
\hline 2 & $\begin{array}{l}\text { Performance Analysis of Linear } \\
\text { Layer Neural } \\
\text { Networks for Oral Cancer Classifi- } \\
\text { cation [9] }\end{array}$ & $\begin{array}{l}\text { Linear layer } \\
\text { neural net- } \\
\text { work }\end{array}$ & $\begin{array}{l}\text { Oral } \\
\text { cancer }\end{array}$ \\
\hline 3 & $\begin{array}{l}\text { Computer Aided Diagnosis in Digi- } \\
\text { tal Pathology } \\
\text { Application: Review and Perspective } \\
\text { Approach in } \\
\text { Lung Cancer Classification[10] }\end{array}$ & $\begin{array}{l}\text { SVM } \\
\text { CNN } \\
\text { Fuzzy } \\
\text { means c- } \\
\text { CAD-FCM }\end{array}$ & $\begin{array}{l}\text { Lung } \\
\text { tissue }\end{array}$ \\
\hline 4 & $\begin{array}{l}\text { Curvelet And PNN Classifier Based } \\
\text { Approach For Early Detection And } \\
\text { Classification Of Breast Cancer In } \\
\text { Digital Mammograms [11] }\end{array}$ & $\begin{array}{l}\text { Curvelet } \\
\text { PNN }\end{array}$ & $\begin{array}{l}\text { Breast } \\
\text { cancer }\end{array}$ \\
\hline 5 & $\begin{array}{l}\text { A Hybrid Approach Based on Deci- } \\
\text { sion Trees and Clustering } \\
\text { for Breast Cancer Classification [12] }\end{array}$ & Decision tree & $\begin{array}{l}\text { Breast } \\
\text { cancer }\end{array}$ \\
\hline
\end{tabular}

\section{Proposed work}

The proposed system predicts the arrhythmia patients in the cancer data set by considering the medical data, with 25 different parameters. Basically, cancer has 23 different categories, and in this system the cancer is classified based on age. Multiple algorithms are applied on same data set and predicting the arrhythmia cases among them and predicting the number of ICU admission days based on Apache and SOFA score of the patient. Finally comparing the accuracy of the result of all the different algorithms.

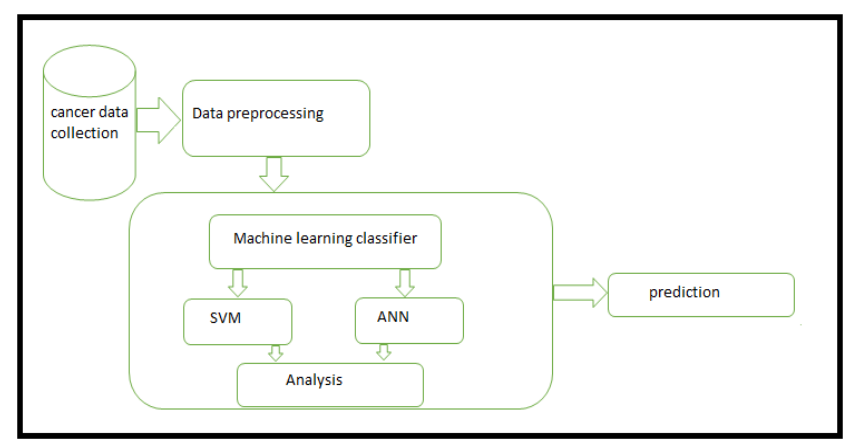

Figure 4.1 Block diagram of Proposed System

\subsection{Methodology}

Step 1: Collection of data

Data is gathered from the hospital with multiple parameter, for further operation need to predict the arrhythmia and number of ICU admission days based on Apache and SOFA score.

Step 2: Gathering and Normalizing data

Data normalization is applying on gathering data and make it in form which is required as an input to the SVM algorithm.

Step 3: Apply Machine Learning algorithms

On normalized data apply Machine Learning algorithms and do analysis of each algorithms results. In that use SVM algorithm methods on dataset.

Step 4: Prediction

From applying machine learning algorithms on data system give the prediction of arrhythmia cases in the data set.
Step 5: Accuracy evaluation of Algorithms

The system gives the accuracy evaluation of two algorithms. In that it shows which gives the better accuracy of the prediction of arrhythmia cases.

\section{Conclusion}

Cancer is the one of the common disease in India and worldwide. Because of cancer patients are getting many other diseases and admitting to ICU (Intensive care unit). Treating and managing the critically ill patients is the difficult task for intensivist. When the patient is in critical stage its doctor's duty to explain their condition to their parents or relatives. To explain those condition, they should do analysis of the patient data. So, analysing the cancer patient data will be helpful for intensivist.

Arrhythmia is one of the dangerous diseases. In critically ill cancer patient arrhythmia can be found. Predicting arrhythmia will be helpful to intensivist to guide patient's relative for the further treatment. Age wise classification will be helpful to predict the arrhythmia cases in early age to old age.

Critically ill patients are getting admitted to ICU, its doctor's duty to tell approximate days of admission required in ICU. This number of ICU admission days will be depending on Apache and SOFA score of the patient. If we do analysis on Apache vs number of ICU admission days and SOFA score vs number of ICU admission days will be very useful for doctors to predict the patient admission days in intensive care unit.

SVM is popular for its prediction and classification accuracy, and Artificial neural network is also one of the popular tools for prediction and classification. Comparing those to algorithm result will be helpful for improve the accuracy in further analysis study. Testing and training the data set and getting the result and test the unknown dataset will helpful for get the proper accuracy result.

\section{Acknowledgement}

I would like to thank Prof. Sachin Bojewar for his guidance and constant supervision for providing necessary information and for his support in carrying out this paper work.

\section{References}

[1] E. Torti, C. Cividini, A. Gatti, G. Danese, F. Leporati, H. Fabelo, S. Ortega, G. M. Callicò "The HELICoiD project: parallel SVM for brain cancer classification" 2017 Euromicro Conference on Digital System Design

[2] Devi Nurtiyasari, Dedi Rosadi and Abdurakhman "The Application Of Wavelet Recurrent Neural Network/or Lung Cancer Classification" 2017 3rd International Conference on Science and Technology - Computer (1CST)

[3] Asad Azemi, Senior Member IEEE, Vahid R. Sabzevari, Morteza Khademi, Hossein Gholizade,

[4] Arman Kiani, and Zeinab S. Dastgheib "Intelligent Arrhythmia Detection and Classification Using ICA" Proceedings of the 28th IEEE EMBS Annual International Conference New York City, USA, Aug 30-Sept 3, 2006

[5] Jiankang Liu Institute of Electronics Chinese Academy of Sciences, Beijing, China, XianXiang Chen Institute of Electronics Chinese Academy of Sciences, Beijing, China, Zhen Fang Institute of Electronics Chinese Academy of Sciences, Beijing, China "ICU mortality prediction using modified cost-sensitive PCA and chaos PSO" 2017 IEEE 19th International Conference on e-Health Networking, Applications and Services (Healthcom)

[6] Pablo Guillen, Jerry Ebalunode "Cancer Classification Based on Microarray Gene Expression Data Using Deep Learning” 2016 International Conference on Computational Science and Computational Intelligence.

[7] Rajalaxmi Hegde, Dr. Seema. S “Aspect Based Feature Extraction and Sentiment Classification of Review Data sets using Incremental Machine learning Algorithm" 3rd International Conference on Ad- 
vances in Electrical, Electronics, Information, Communication and Bio-Informatics (AEEICB17)

[8] Mourad Adnane, Adel Belouchrani "On the use of Auto-Regressive Modeling for Arrhythmia Detection” 2016 24th European Signal Processing Conference (EUSIPCO)

[9] https://www.uptodate.com/contents/reentry-and-the-developmentof-cardiac-arrhythmias Accessed 28 February, 2018

[10] Sunil Kumar Prabhakar, Harikumar Rajaguru "Performance Analysis of Linear Layer Neural Networks for Oral Cancer Classification" 2017 6th ICT International Student Project Conference (ICT ISPC)

[11] Abbas K. AlZubaidi, Fahad B. Sideseq, Ahmed Faeq, Mena Basil "Computer Aided Diagnosis in Digital Pathology Application: Review and Perspective Approach in Lung Cancer Classification" Annual Conference on New Trends in Information \& Communications Technology Applications-(NTICT'2017) 7 - 9 March 2017

[12] Anu Appukuttan ,Sindhu L" Curvelet And PNN Classifier Based Approach For Early Detection And Classification Of Breast Cancer In Digital Mammograms" 2016 International Conference on Inventive Computation Technologies (ICICT)

[13] Hind Elouedi, Walid Meliani, Zied Elouedi, Nahla Ben Amor “A Hybrid Approach Based on Decision Trees and Clustering for Breast Cancer Classification" 2014 6th International Conference of Soft Computing and Pattern Recognition (SoCPaR) 